\title{
ВОЗМОЖНОСТИ ПРИМЕНЕНИЯ СD4+-РЕГУЛЯТОРНЫХ Т-КЛЕТОК В ТЕРАПИИ АУТОИММУННЫХ ЗАБОЛЕВАНИЙ
}

\author{
А. В. Чуров ${ }^{1}$, А. И. Сюткина², К. Ы. Мамашов ${ }^{3}$, Е. К. Олейник ${ }^{1}$ \\ 1 Федеральный исследовательский центр «Карельский научный центр Российской академии наук», Петрозаводск, Россия \\ 2 Пермская государственная фармацевтическая академия, Пермь, Россия \\ ${ }^{3}$ Кемеровский государственный медицинский университет, Кемерово, Россия
}

\begin{abstract}
На сегодняшний день достигнуты значительные успехи в терапии аутоиммунных патологий, однако существующие методы сопряжены с риском возникновения тяжелых побочных эффектов. Применение Treg-клеток, на наш взгляд, представляет значительную перспективу в терапии состояний, связанных с нарушением аутотолерантности. В работе дана оценка возможных трудностей использования клинического подхода на основе Ттеg-клеток и предложены пути решения научно-технических задач, возникающих при применении Treg в терапии аутоиммунных заболеваний.

Ключевые слова: регуляторные Т-клетки, FOXP3, аутоиммунное заболевание, иммунотерапия, клеточная терапия, CAR-Treg-терапия, CRISPR/Cas9

Финансирование: финансовое обеспечение исследований было осуществлено из средств федерального бюджета на выполнение государственного задания КарНЦ РАН, № 0218-2019-0083 (по теме «Изменение транскрипционных программ дифференцировки регуляторных Т-клеток при иммуновос-
\end{abstract} палительных и онкологических патологиях»). Спонсор публикации ООО «ПРАЙМ ПЕЙПЕРС».

Благодарности: авторы признательны Центру высокоточного редактирования и генетических технологий для биомедицины (Москва) за консультации в рамках подготовки статьи.

Информация о вкладе авторов: А. В. Чуров - дизайн статьи, анализ литературы, подготовка рукописи и финального варианта статьи; А. И. Сюткина дизайн статьи, основной вклад в анализ литературы, подготовку рукописи и финального варианта статьи; К. Ы. Мамашов - дизайн статьи, анализ литературы, участие в подготовке рукописи и финального варианта статьи; Е. К. Олейник - анализ литературы, участие в подготовке рукописи и финального варианта статьи.

$\triangle$ Для корреспонденции: Алексей Викторович Чуров ул. Пушкинская, д. 11, г. Петрозаводск, 186910; achurou@yandex.ru

Статья получена: 25.11.2019 Статья принята к печати: 09.12.2019 Опубликована онлайн: 18.12.2019

DOI: $10.24075 /$ vrgmu.2019.082

\section{THE POTENTIAL OF CD4+ REGULATORY T CELLS FOR THE THERAPY OF AUTOIMMUNE DISEASES}

Churov $A V^{1} \otimes$, Siutkina $A l^{2}$, Mamashov $\mathrm{KY}^{3}$, Oleinik $E K^{1}$

${ }^{1}$ Karelian Research Centre of the Russian Academy of Sciences, Petrozavodsk, Russia

2 Perm State Pharmaceutical Academy, Perm, Russia

${ }^{3}$ Kemerovo State Medical University, Kemerovo, Russia

Despite the considerable progress in the therapy of autoimmune pathologies, the existing methods are associated with the risk of serious adverse events. We think that regulatory $T$ cells hold great promise for the therapy of disorders caused by a breakdown in immunological self-tolerance. This article aims at estimating the possible challenges facing Treg-based clinical approaches and offers solutions to the technical issues associated with the use of these cells in the therapy of autoimmune diseases.

Keywords: regulatory T cells, FOXP3, autoimmune disease, immunotherapy, cell therapy, CAR-Treg therapy, CRISPR/Cas9

Funding: the study was carried out under state order for Karelian Research Centre (ID 0218-2019-0083; Modification of transcription programs of regulatory $T$ cell differentiation in immunoinflammatory diseases and cancer). Its publication was sponsored by Prime Papers LLC.

Acknowledgements: the authors thank the Center for Precision Genome Editing and Genetic Technologies for Biomedicine (Moscow) for consultations.

Author contribution: Churov AV — article design, literature analysis, preparation of the manuscript draft and its final version; Syutkina Al — article design, the major contribution to literature analysis, preparation of the manuscript draft and its final version; Mamashov KY — article design, literature analysis, preparation of the manuscript draft and its final version; Oleinik EK — literature analysis, preparation of the manuscript draft and its final version.

$\bowtie$ Correspondence should be addressed: Alexey V. Churov

Pushkinskaya, 11, Petrozavodsk, 186910; achurou@yandex.ru

Received: 25.11.2019 Accepted: 09.12.2019 Published online: 18.12.2019

DOI: 10.24075/brsmu.2019.082

Понятие аутоиммунная патология подразумевает под собой группу заболеваний. В их основе лежит дисбаланс в работе иммунной системы и механизмах поддержания аутотолерантности, приводящий к развитию комплекса иммунных реакций против собственных тканей организма.

В настоящее время терапией выбора при аутоиммунных заболеваниях является главным образом использование иммунодепрессантов, синтетических базисных противовоспалительных препаратов (DMARD), a также генно-инженерных препаратов. Однако применение таких средств сопряжено с риском серьезных побочных эффектов. Регуляторные Т-клетки (Treg) препятствуют развитию иммунных реакций к аутоантигенам, контролируют развитие патологического воспаления и, как следствие, аутоиммунных заболеваний, непосредственно участвуя в индукции и поддержании периферической толерантности. Treg-клетки составляют 3-5\% от общего числа $\mathrm{CD}_{4}^{+}$ Т-лимфоцитов в периферическом русле и могут подавлять активацию, пролиферацию и эффекторные функции $\mathrm{CD}^{+}{ }^{+}$и $\mathrm{CD} 8^{+-}$-лиммфоцитов, NK-клеток, В-клеток, антигенпрезентирующих клеток (АПК) [1]. У пациентов с аутоиммунными патологиями можно наблюдать изменение количества Treg-клеток, при этом нередко их функция ослаблена или нарушена [2, 3]. Экспериментальные исследования, проведенные на мышиных моделях аутоиммунных заболеваний, показали, что адоптивная 
терапия Treg-клетками, полученными от здоровых особей, обладает хорошим терапевтическим эффектом [4]. Благодаря позитивным результатам доклинических исследований, Treg-лимфоциты стали рассматривать в качестве перспективного подхода к лечению аутоиммунных заболеваний.

С учетом текущего состояния исследований и ограничения современных методов лечения необходима разработка новых высокоспецифичных подходов для эффективной и безопасной терапии аутоиммунных патологий. Мы предполагаем, что клинические решения на основе Treg-клеток могут занять центральное место в комплексной терапии аутоиммунных заболеваний при условии решения ряда технических задач.

В работе отражено мнение о перспективах применения Treg-клеток в качестве инструмента для терапии аутоиммунных заболеваний на основании имеющихся результатов научных и клинических исследований, дана оценка возможных трудностей, возникающих при использовании данного подхода, и предложены потенциальные пути решения научно-технических проблем в данной области.

\section{Регуляторные Т-клетки и механизмы супрессорного действия}

Ключевыми маркерами Treg-клеток служат мембранный антиген CD25 и внутриклеточный транскрипционный фактор FOXP3. Экспрессия гена FOXP3 необходима для реализации супрессорной функции Treg-лимфоцитов [5]. Маркер CD25 представляет собой $\alpha$-цепь IL2, его экспрессия коррелирует с пролиферацией и дифференцировкой Treg [6].

Дифференцировка Treg-клеток происходит в тимусе и на периферии. Из тимуса Treg расселяются в кровь и периферические органы лимфоидной системы в течение первых 2-3 дней жизни [7]. На периферии Т-клетки с фенотипом CD4+FOXP3- также могут подвергаться дифференцировке с образованием индуцированных Treg-клеток, отличающихся высокой экспрессией FOXP3 (CD4 $\left.{ }^{+} \mathrm{CD} 25^{+} \mathrm{FOXP3}^{+}\right)$.

Супрессорное действие Treg на иммуноциты может быть реализовано напрямую при межклеточном контакте или опосредованно, в результате секреции биологически активных молекул. Можно выделить четыре основных механизма: прямой цитотоксический эффект (при участии PD-1/PD-L1, OX40/OX40L и гранзимов B), изменение метаболизма клеток-мишеней (CD25, CD39, триптофан), секреция ингибирующих цитокинов (IL10, TGF $\beta$ и IL35), а также супрессия AПK (CD80 и CD86/CTLA-4) [8].

\section{Клинические испытания иммунотерапии на основе Treg-клеток}

Клинические испытания иммунотерапии на основе Treg-клеток при аутоиммунных заболеваниях начались менее 10 лет назад. Целью испытаний стала проверка технических аспектов при инфузии Treg, а также оценка безопасности и эффективности метода. В клинических испытаниях применяют общий подход: сортировка Treg-клеток, поликлональная экспансия Treg, подбор дозы и проведение инъекций Treg-лимфоцитов пациентам. Однако данный подход не учитывает функциональный статус клеток. Общей проблемой при использовании Treg остается отсутствие стабильной экспрессии FOXP3 и стойкой супрессорной активности, что во многих случаях обусловливает невысокую эффективность применения данной технологии для клеточной терапии.

Согласно открытому многоцентровому исследованию CATS1, опубликованному в 2012 г. [9], на этапе I/Ila, включающем 20 пациентов из четырех групп с активной и симптоматической ресрактерной болезнью Крона, была показана безопасность инфузии клеток. В феврале 2018 г. было начато двойное слепое плацебо-контролируемое исследование NCT03185000 (TRIBUTE), оценивающее инфузию Treg с фенотипом CD4 ${ }^{+} \mathrm{CD} 25^{+} \mathrm{CD} 127^{\text {low }} \mathrm{CD} 45 \mathrm{RA}{ }^{+}$ у пациентов с болезнью Крона, резистентных минимум к двум стандартным схемам терапии.

В целом хорошие результаты получены в ходе применения Treg для иммунотерапии при сахарном диабете 1-го типа (СД1). Клинические испытания стартовали в 2014 г. с рандомизированного исследования фазы I с целью оценки безопасности и целесообразности инфузии аутологичных Treg, полученных ex vivo у пациентов в возрасте 7-18 лет с недавно диагностированным СД1 [10]. По результатам годового исследования не было выявлено сильных побочных эффектов и тем самым подтверждена безопасность лечения. Кроме того, у 8 из 12 пациентов были выявлены признаки клинической ремиссии.

С той же целью в другом исследовании на базе Калифорнийского и Йельского университетов было проведено изучение эффективности и безопасности иммунотерапии у взрослых пациентов с СД1, результаты были опубликованы в 2015 г. [11]. В исследовании приняли участие 14 человек (6 женщин и 8 мужчин в возрасте 18-43 лет), разделенных на четыре группы в зависимости от дозы Treg при инфузии. Для инфузии применяли поликлональные Treg-клетки с фенотипом $\mathrm{CD} 4{ }^{+} \mathrm{CD} 127^{\mathrm{lo} /} \mathrm{CD} 25^{+}$в количестве от $0,05 \times 10^{8}$ до $26 \times 10^{8}$ клеток. По результатам исследования, до $25 \%$ клеток от их пикового уровня сохранялось в периферическом русле реципиентов еще в течение года после адоптивного переноса. При этом не было выявлено никаких инфузионных реакций или побочных эфффектов, связанных с клеточной терапией [11]. Однако данная работа не позволила определить оптимальное количество клеток для инсузии, а также установить, как влияет иммунотерапия на основе Treg на функцию $\beta$-клеток поджелудочной железы. Многоцентровое рандомизированное плацебо-контролируемое двойное слепое клиническое исследование II фразы (NCT02691247) ставит своей целью решить эти вопросы. В настоящее время идут рандомизированные открытые исследования фазы I/II NCT03011021 и NCT02932826 (Центральный Южный университет Чанша), оценивающие инфузию Treg пуповинной крови наряду с терапией лираглутидом и инсулином у взрослых и пожилых пациентов с аутоиммунным диабетом.

Кроме того, начаты открытые исследования по инфузии аутологичных поликлональных Treg у пациентов с временной активной кожной пузырчаткой (NCT03239470) и аутоиммунным гепатитом (NCT02704338).

\section{Перспективы и трудности, связанные с применением Treg-клеток в иммунотерапии}

Принципиальная возможность осуществления инфузии Treg-клеток пациентам с различными аутоиммунными патологиями и относительная безопасность данного подхода были продемонстрированы в ходе пилотных 
клинических исследований. На дальнейших этапах представляет интерес адаптировать применяемую у пациента в текущий момент иммуносупрессивную терапию к инсузиям Treg, так как показано, что некоторые иммунодепрессанты дозозависимо влияют на свойства Treg-клеток и могут снижать эффективность клеточной терапии [12]. Наиболее перспективным подходом представляется применение Treg в комбинации с другими методами лечения, необходимыми в каждом конкретном случае.

Одно из основных препятствий для успешного применения Treg в иммунотерапии - получение стабильной фракции Treg-клеток, проявляющих стойкую иммуносупрессорную активность. Существующие методы получения Treg основаны на экспансии лимфоцитов ех vivo. При этом источником Treg служат клетки периферической крови, которые неоднородны и в значительной степени представлены клетками с индуцированной экспрессией FOXP3.

Другой не менее важной проблемой является повышение специфичности терапии. Функциональная активность Treg по своей природе антигенспецифична. В присутствии антигена происходят активация Treg и значительное увеличение экспрессии мембранных ингибирующих молекул, которые обусловливают супрессорное действие на клетки-мишени.

Эта зависимость была продемонстрирована in vivo на примере CTLA-4 - ключевой ингибирующей молекулы Treg-клеток. CTLA-4 конститутивно экспрессируется как Treg, так и Т-эффекторами [13]. Treg контролируют активацию Т-эффекторов, ограничивая их доступ к костимулирующим молекулам CD80/86 на мембране AПК. CTLA-4 конкурентно блокирует CD80/86 на AПК, а также может связывать и перемещать CD80/86 внутрь клетки в результате трансэндоцитоза. Таким образом, Treg-клетки регулируют фенотип АПК и эфффективно ограничивают CD28зависимую активацию Т-эффекторов. Конкурентный эффект достигается за счет того, что экспрессия CTLA-4 на антигенспецифичных Treg-клетках значительно выше, чем на Т-клетках. Триггером к захвату CD80/86 Tregклетками является презентация аутоантигена [13].

Клинические испытания, о которых говорилось выше, как правило, включают применение инфузии поликлональных Treg-клеток с неизвестной антигенной специфичностью. Большая часть клонов в составе фьюзов будет неэффективна в отношении супрессии клетокэффекторов и в подавлении аутоиммунных реакций. Кроме того, при использовании поликлональных лимфоцитов сохранаяется высокий риск возникновения побочных эффектов, таких как системная иммуносупрессия и реактивация инфекций. Таким образом, клинические подходы на основе поликлональных Treg заведомо слабы.

В последнее десятилетие были разработаны новые высокоэффективные подходы лечения некоторых патологий на основе адоптивной клеточной иммунотерапии, которые могут позволить преодолеть вышеуказанные трудности в применении Treg для лечения аутоиммунных заболеваний. K таким подходам относят конструкции на основе эфффекторных Т-клеток для экспрессии высокоспецисричных химерных антигенных рецепторов (CARs), а также методы редактирования генома, в частности CRISPR/Cas9.

CAR-модифицированные T-клетки (CAR-T) применяют в иммунотерапии некоторых форм рака. Однако метод достаточно эффективен и в случае с Treg-клетками (CARTreg). CAR-Treg-технология имеет огромный потенциал: благодаря антигенспецифичности CAR-Treg позволяют повысить эффективность лечения при минимальных побочных эфффектах [14]. В сравнении с использованием в терапии поликлональных Treg, CAR-Treg способны специфично связываться с определенным белком на мембране клеток-мишеней. Среди недостатков технологии можно отметить высокую сложность получения CAR-Treg со стабильной супрессорной активностью. Кроме того, существует опасность возникновения так называемого цитокинового шторма и нейротоксичности, как и в случае с противоопухолевыми CAR-T-клетками, что оказывается серьезным препятствием для использования CAR-Treg в клинике. Более того, препятствием для применения CAR-Treg являются идентификация и выбор мишеней для таргетной терапии, что особенно важно в случае с аутоиммунными заболеваниями в связи с особенностями их патогенеза и недостатком знаний об их этиологии.

Один из вышеуказанных недостатков, а именно получение стабильной фракции клеток, могут помочь устранить технологии на основе CRISPR/Cas9 (clustered, regularly interspaced, short palindromic repeats / CRISPRassociated protein) или их более продвинутых аналогов. Первые доклинические исследования уже показали многообещающие результаты применения CRISPR/Cas9 в иммунотерапии. Предпринимаются попытки модификации функциональной активности Treg, в плане как стабилизации их активности, так и повышения уровня активности Treg. Известно, что одной из основных проблем применения Treg остается поддержание жизнеспособности клеток ех vivo, которая напрямую зависит от стабильности экспрессии гена FOXP3. В этой связи предлагают использовать технологию CRISPR/Cas9 для редактирования генов, участвующих в регуляции транскрипционных программ, контролирующих дифференцировку Treg-клеток и уровень экспрессии FOXP3. CRISPR/Cas9 обладает высокой специфичностью благодаря малым РНК: они способны направлять нуклеазу Cas9 точно в целевой фрагмент генома [15]. В случае с Treg-клетками и FОХРЗ технология представляет интерес возможностью редактировать эпигеном. В первичных Т-клетках у мышей было показано, что используя модификацию базовой технологии, включающую мутантный Cas9 без эндонуклеазной активности (dead Cas9; CRISPR/ dCas9), можно индуцировать заданные эпигенетические изменения, способствующие стабилизации экспрессии гена Foxp3 [16].

\section{ВЫВОДЫ}

Первые результаты клинических исследований продемонстрировали относительную безопасность применения Treg-клеток для лечения аутоиммунных патологий. Многообещающим стало исследование эфффективности подхода, основанного на применении поликлональных Treg-лимфоцитов у пациентов с болезнью Крона и диабетом 1-го типа, а также для некоторых других аутоиммунных заболеваний. Однако большинство клинических исследований безопасности применения Treg были проведены на малых выборках, а сам подход имеет ряд серьезных недостатков, прежде всего низкую антигенную специфичность. Дальнейшие усилия должны быть направлены на нерешенные вопросы в отношении технологии адоптивной терапии Treg-клеток: поиск эффективной дозы Treg для инфузии; повышение специфичности метода за счет идентификации мишеней 
для таргетной клеточной иммунотерапии и использования фьюзов антигенспецифичных Treg; достижение стабильной супрессорной активности Treg и стойкого эффекта от клеточной терапии у пациентов с аутоиммунными заболеваниями.

\section{Литература}

1. Sakaguchi S, Miyara M, Costantino CM, Hafler DA. FOXP3+ regulatory $T$ cells in the human immune system. Nat Rev Immunol. 2010; 10 (7): 490-500. DOI:10.1038/nri2785.

2. Venken K, Hellings N, Liblau R, Stinissen P. Disturbed regulatory T cell homeostasis in multiple sclerosis. Trends Mol Med. 2010; 16 (2): 58-68. DOI: 10.1016/j.molmed.2009.12.003.

3. Кравченко П. Н., Жулай Г. Ф., Чуров А. В., Олейник Е. К., Олейник В. М., Барышева О. Ю. и др. Субпопуляции регуляторных Т-лимфоцитов в периферической крови больных ревматоидным артритом. Вестник РАМН. 2016; 71 (2): 148-53. DOI: 10.15690/vramn656.

4. Miyara M, Gorochov G, Ehrenstein M, Musset L, Sakaguchi S, Amoura Z. Human FoxP3 ${ }^{+}$regulatory $T$ cells in systemic autoimmune diseases. Autoimmunity Reviews. 2011; 10 (12): 744-55. DOI:10.1016/j.autrev.2011.05.004.

5. Fontenot J, Gavin M, Rudensky A. Foxp3 programs the development and function of $\mathrm{CD} 4{ }^{+} \mathrm{CD} 25^{+}$regulatory $\mathrm{T}$ cells. Nature Immunology. 2003; 4 (4): 330-36. DOI: 10.1038/ni904.

6. Nazzal, Gradolatto, Truffault, Bismuth, Berrih-Aknin. Human thymus medullary epithelial cells promote regulatory T-cell generation by stimulating interleukin-2 production via ICOS ligand. Cell Death Dis. 2014; (5): e1420. DOI:10.1038/cddis.2014.37.

7. Famili F, Wiekmeijer A-S, Staal F. The development 719 of T cells from stem cells in mice and humans. Future Science OA. 2017; (3): FSO186. DOI:10.4155/fsoa-2016-0095.

8. Christoffersson G, von Herrath M. Regulatory Immune Mechanisms beyond Regulatory T Cells. Trends in Immunology. 2019; 40 (6): 482-91. DOI:10.1016/j.it.2019.04.005.

9. Desreumaux P, Foussat A, Allez M, Beaugerie L, Hébuterne X, Bounnik $Y$, et al. Safety and efficacy of antigen-specific regulatory T-cell therapy for patients with refractory Crohn's disease. Gastroenterology. 2012; (143): 1207-17. DOI:10.1053/j.

\section{References}

1. Sakaguchi S, Miyara M, Costantino CM, Hafler DA. FOXP3+ regulatory $T$ cells in the human immune system. Nat Rev Immunol. 2010; 10 (7): 490-500. DOI:10.1038/nri2785.

2. Venken K, Hellings N, Liblau R, Stinissen P. Disturbed regulatory T cell homeostasis in multiple sclerosis. Trends Mol Med. 2010; 16 (2): 58-68. DOI: 10.1016/j.molmed.2009.12.003.

3. Kravchenko PN, Zhulai GA, Churov AV, Oleinik EK, Oleinik VM, Barysheva OY, et al. Subpopulations of regulatory T-lymphocytes in the peripheral blood of patients with rheumatoid arthritis. Vestnik Rossiiskoi Akademii Meditsinskikh Nauk. 2016; 71 (2): 148-153. DOI: 10.15690/vramn656.

4. Miyara M, Gorochov G, Ehrenstein M, Musset L, Sakaguchi S, Amoura Z. Human FoxP3 ${ }^{+}$regulatory $T$ cells in systemic autoimmune diseases. Autoimmunity Reviews. 2011; 10 (12): 744-55. DOI:10.1016/j.autrev.2011.05.004.

5. Fontenot J, Gavin M, Rudensky A. Foxp3 programs the development and function of $\mathrm{CD}^{+}{ }^{+} \mathrm{CD} 25^{+}$regulatory $\mathrm{T}$ cells. Nature Immunology. 2003; 4 (4): 330-36. DOI: 10.1038/ni904.

6. Nazzal, Gradolatto, Truffault, Bismuth, Berrih-Aknin. Human thymus medullary epithelial cells promote regulatory T-cell generation by stimulating interleukin-2 production via ICOS ligand. Cell Death Dis. 2014; (5): e1420. DOI:10.1038/cddis.2014.37.

7. Famili F, Wiekmeijer A-S, Staal F. The development 719 of T cells from stem cells in mice and humans. Future Science OA. 2017; (3): FSO186. DOI:10.4155/fsoa-2016-0095.

8. Christoffersson G, von Herrath M. Regulatory Immune Mechanisms beyond Regulatory $\mathrm{T}$ Cells. Trends in Immunology. 2019; 40 (6): 482-91. DOI:10.1016/j.it.2019.04.005. gastro.2012.07.116.

10. Marek-Trzonkowska N, Myśliwiec M, Dobyszuk A, Grabowska M, Derkowska I, et al. Therapy of type 1 diabetes with CD4(+) CD25(high)CD127-regulatory $\mathrm{T}$ cells prolongs survival of pancreatic islets - results of one year follow-up. Clinical immunology (Orlando, Fla). 2014; (153): 23-30. DOl:10.1016/j. clim.2014.03.016.

11. Bluestone JA, Buckner JH, Fitch M, Gitelman SE, Gupta S, Hellerstein MK, et al. Type 1 diabetes immunotherapy using polyclonal regulatory $\mathrm{T}$ cells. Science translational medicine. 2016; (7): 315ra189. DOI:10.1126/scitranslmed.aad4134.

12. Scottà C, Fanelli G, Hoong SJ, Romano M, Lamperti EN, Sukthankar $\mathrm{M}$, et al. Impact of immunosuppressive drugs on the therapeutic efficacy of ex vivo expanded human regulatory T cells. Haematologica. 2016; (101): 91-100. DOI:10.3324/ haematol.2015.128934.

13. Ovcinnikovs V, Ross EM, Petersone L, Edner NM, Heuts F, Ntavli E. CTLA-4-mediated transendocytosis of costimulatory molecules primarily targets migratory dendritic cells. Science Immunology. 2019; 4 (35): eaaw0902. DOI:10.1126/sciimmunol.aaw0902.

14. Arpaia N, Campbell C, Fan X, Dikiy S, Veeken J van der, deRoos P, et al. Metabolites produced by commensal bacteria promote peripheral regulatory T-cell generation. Nature. 2013; (504): 4515. DOI:10.1038/nature12726.

15. Jinek M, Chylinski K, Fonfara I, Hauer M, Doudna JA, Charpentier E. A Programmable Dual-RNA-Guided DNA Endonuclease in Adaptive Bacterial Immunity. Science. 2012; 337 (6096): 816-21. DOI:10.1126/science.1225829.

16. Okada M, Kanamori M, Someya K, Nakatsukasa H, Yoshimura A. Stabilization of Foxp3 expression by CRISPR-dCas9-based epigenome editing in mouse primary $\mathrm{T}$ cells. Epigenetics Chromatin. 2017; (10): 24. DOI: 10.1186/s13072-017-0129-1.

9. Desreumaux P, Foussat A, Allez M, Beaugerie L, Hébuterne X, Bouhnik $Y$, et al. Safety and efficacy of antigen-specific regulatory T-cell therapy for patients with refractory Crohn's disease. Gastroenterology. 2012; (143): 1207-17. DOI:10.1053/j. gastro.2012.07.116.

10. Marek-Trzonkowska N, Myśliwiec M, Dobyszuk A, Grabowska M, Derkowska I, et al. Therapy of type 1 diabetes with CD4(+) CD25(high)CD127-regulatory $\mathrm{T}$ cells prolongs survival of pancreatic islets - results of one year follow-up. Clinical immunology (Orlando, Fla). 2014; (153): 23-30. DOI:10.1016/j. clim.2014.03.016.

11. Bluestone JA, Buckner JH, Fitch M, Gitelman SE, Gupta S, Hellerstein MK, et al. Type 1 diabetes immunotherapy using polyclonal regulatory $T$ cells. Science translational medicine. 2016; (7): 315ra189. DOI:10.1126/scitranslmed.aad4134.

12. Scottà C, Fanelli G, Hoong SJ, Romano M, Lamperti EN, Sukthankar $\mathrm{M}$, et al. Impact of immunosuppressive drugs on the therapeutic efficacy of ex vivo expanded human regulatory T cells. Haematologica. 2016; (101): 91-100. DOI:10.3324/ haematol.2015.128934.

13. Ovcinnikovs V, Ross EM, Petersone L, Edner NM, Heuts F, Ntavli E. CTLA-4-mediated transendocytosis of costimulatory molecules primarily targets migratory dendritic cells. Science Immunology. 2019; 4 (35): eaaw0902. DOI:10.1126/sciimmunol.aaw0902.

14. Arpaia N, Campbell C, Fan X, Dikiy S, Veeken J van der, deRoos P, et al. Metabolites produced by commensal bacteria promote peripheral regulatory T-cell generation. Nature. 2013; (504): 4515. DOI:10.1038/nature12726. 


\section{МНЕНИЕ І ИММУНОЛОГИЯ}

15. Jinek M, Chylinski K, Fonfara I, Hauer M, Doudna JA, Charpentier E. A Programmable Dual-RNA-Guided DNA Endonuclease in Adaptive Bacterial Immunity. Science. 2012; 337 (6096): 816-21. DOI:10.1126/science.1225829.
16. Okada M, Kanamori M, Someya K, Nakatsukasa H, Yoshimura A. Stabilization of Foxp3 expression by CRISPR-dCas9-based epigenome editing in mouse primary $\mathrm{T}$ cells. Epigenetics Chromatin. 2017; (10): 24. DOI: 10.1186/s13072-017-0129-1. 